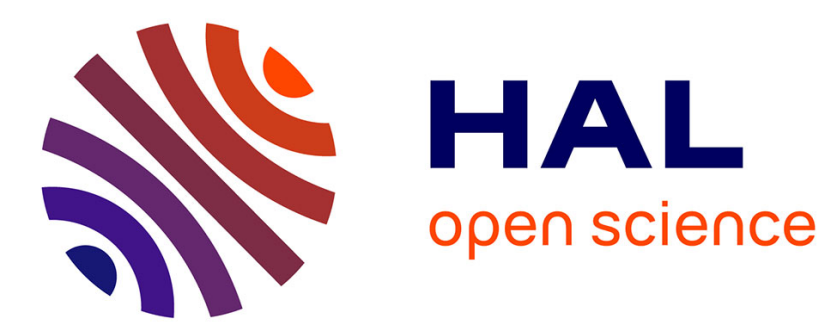

\title{
La géochimie isotopique, le thermomètre des climats du passé \\ Elsa Cortijo
}

\section{To cite this version:}

Elsa Cortijo. La géochimie isotopique, le thermomètre des climats du passé. Clefs CEA, 2009, (Terre et Environnement), 57, pp.17-20. hal-03322845

\section{HAL Id: hal-03322845 \\ https://hal.science/hal-03322845}

Submitted on 19 Aug 2021

HAL is a multi-disciplinary open access archive for the deposit and dissemination of scientific research documents, whether they are published or not. The documents may come from teaching and research institutions in France or abroad, or from public or private research centers.
L'archive ouverte pluridisciplinaire HAL, est destinée au dépôt et à la diffusion de documents scientifiques de niveau recherche, publiés ou non, émanant des établissements d'enseignement et de recherche français ou étrangers, des laboratoires publics ou privés. 


\title{
La géochimie isotopique, le thermomètre des climats du passé
}

\begin{abstract}
Pour reconstruire les changements climatiques passés, l'analyse de la composition isotopique des glaces polaires et des sédiments marins, véritables archives naturelles, permet de recueillir des informations précieuses sur les variations de température et sur la circulation des océans au cours des temps géologiques. Outil incontournable, la géochimie isotopique joue un rôle majeur dans la compréhension des mécanismes climatiques.
\end{abstract}
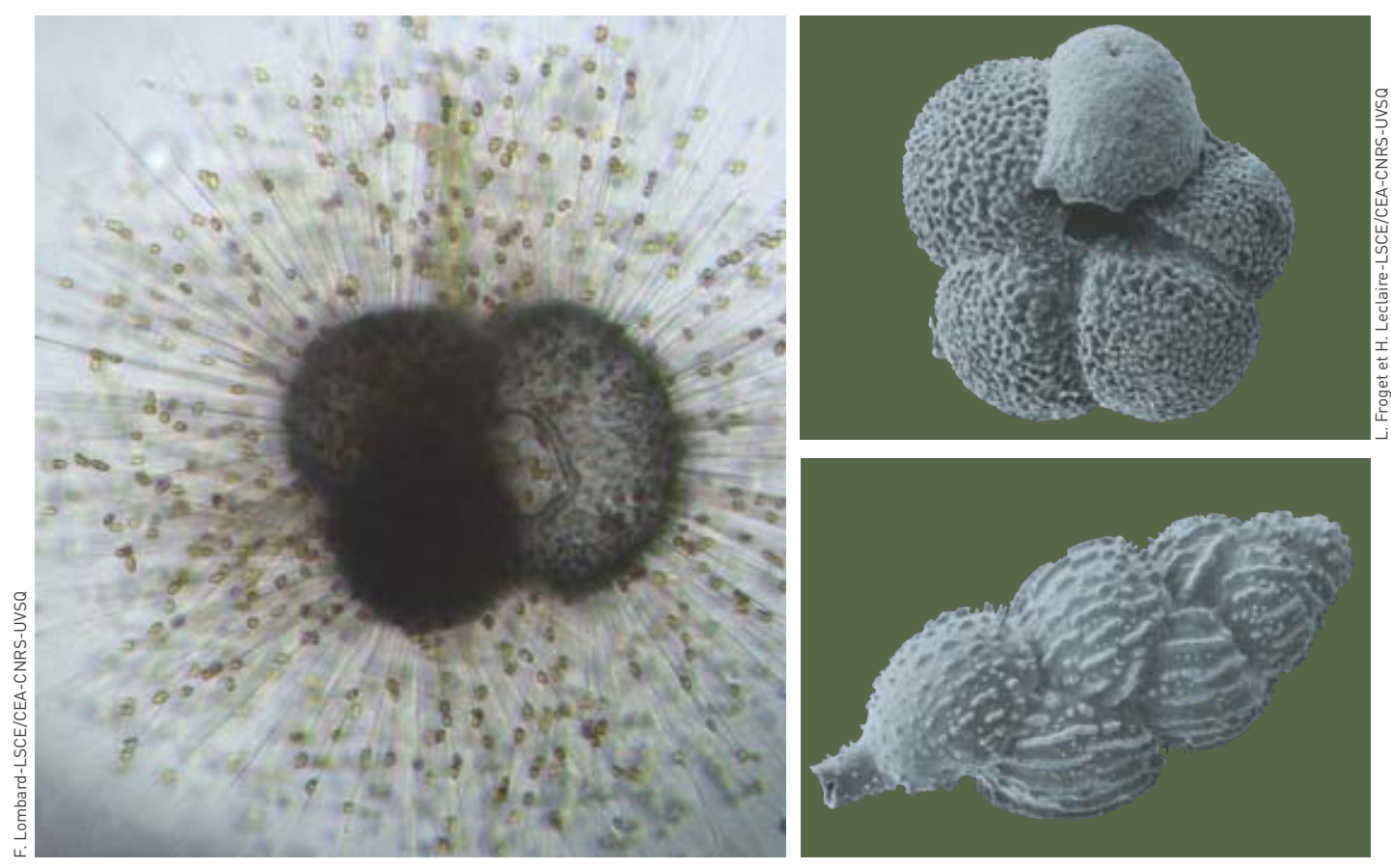

Foraminifère vivant (à gauche) et coquilles de foraminifères (à droite). Lorsque le foraminifère meurt, seule la coquille carbonatée, ou test, sédimente et est préservée dans les sédiments marins. Ce sont ces tests de foraminifères qui sont triés et analysés en spectrométrie de masse pour reconstruire les variations de la composition isotopique de l'oxygène.

es changements climatiques sont un sujet d'actua-lité depuis quelques années, les hivers sans neige succédant aux étés caniculaires, et les nombreux dérèglements climatiques suscitant des polémiques médiatiques. Pour comprendre quels sont les mécanismes gouvernant le climat de la Terre et replacer dans le temps les événements climatiques que nous vivons, les scientifiques analysent les enregistrements météorologiques obtenus par satellites ou par des mesures de terrain. Cependant, la période de temps couverte, de l'ordre de 150 ans pour les réseaux météorologiques, n'autorise pas l'étude des mécanismes dont les constantes de temps sont plus longues.

Dans ce contexte, la paléoclimatologie offre la possibilité de remonter sur plusieurs dizaines, centaines, milliers, voire millions, d'années. Ce sont ces études qui ont permis de mettre en évidence la forte variabilité temporelle du climat avec, en quelques milliers d'années, l'alternance entre des périodes chaudes, comparables à celle dans laquelle l'agriculture néolithique s'est développée, et des périodes glaciaires, au cours desquelles les hautes latitudes de l'hémisphère Nord étaient recouvertes d'épaisses calottes de glace. Mais comment reconstitue-t-on les climats du passé? La géochimie des isotopes stables de l'oxygène et du carbone joue un rôle de tout premier plan. Les isotopes de l'oxygène seront ici plus spécifiquement examinés.

Les isotopes de l'oxygène, indicateurs climatiques

L'oxygène est présent dans l'air que nous respirons ou dans l'eau qui nous entoure sous la forme de plusieurs isotopes de masses respectives 16,17 ou 18 , selon le nombre de neutrons compris dans le noyau. Ces isotopes partagent les mêmes propriétés chimiques mais diffèrent par les propriétés physiques liées à leurs masses. Le rapport ${ }^{18} \mathrm{O} /{ }^{16} \mathrm{O}$ sur Terre de n'importe quel corps renfermant de l'oxygène va être proche de 0,2\%, mais il n'est pas rigoureusement constant. Par exemple, le rapport isotopique d'une masse d'eau donnée va dépendre des phénomènes d'évaporation-précipitation. En effet, l'isotope le plus léger s'évapore plus facilement que l'isotope le plus lourd qui, lui, se condense plus aisément dans les pluies. La composition isotopique de la glace dans les forages polaires ou tropicaux va permettre de suivre au cours du temps l'évolution des processus 


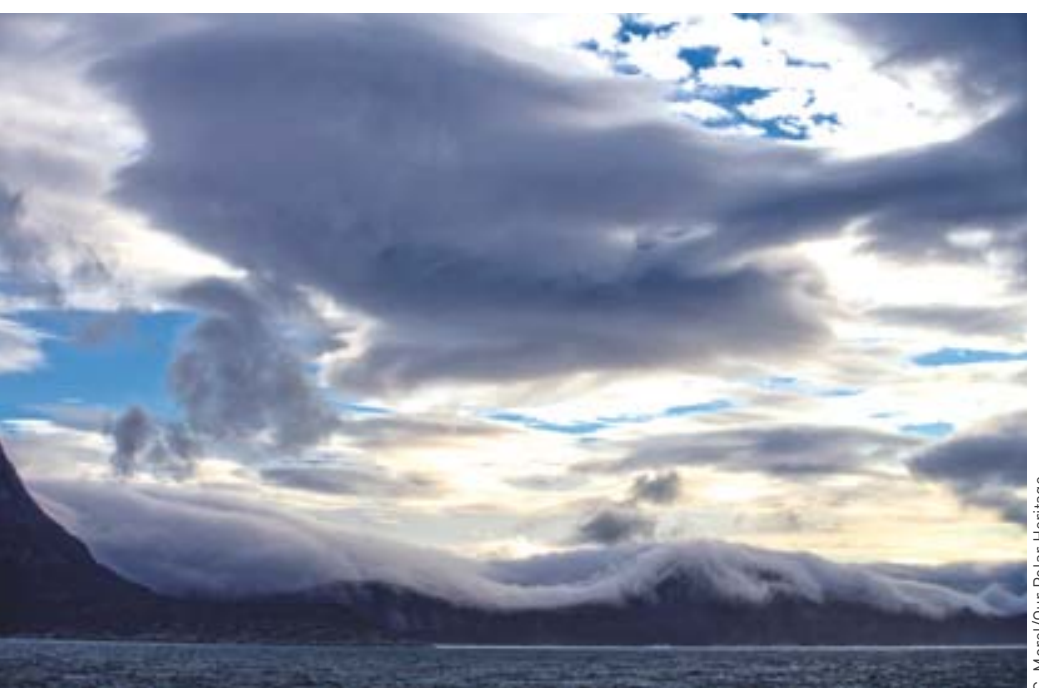

Quand l'eau de l'océan s'évapore aux basses latitudes, un nuage peut se former. Les isotopes légers (molécule d'eau contenant ${ }^{16} 0$ ) s'évaporent plus facilement que les isotopes lourds. Lorsque le nuage se déplace de l'équateur vers les pôles, il va pleuvoir à plusieurs reprises (précipitations). Les isotopes lourds ${ }^{18} 0$ se condensent plus aisément dans les pluies. Le nuage va donc s'appauvrir en isotopes lourds tout au long de son trajet. La neige qui tombera sur les pôles sera par conséquent très "légère" car appauvrie en ${ }^{18} 0$. Cet isotope lourd se retrouvera en revanche dans les océans, particulièrement en période glaciaire. Ici, paysage du Groenland.

physiques qui ont affecté le cycle de l'eau. Autre exemple, un organisme avec une coquille en carbonate de calcium $\left(\mathrm{CaCO}_{3}\right)$ va puiser dans l'eau de mer les ions bicarbonate et les ions calcium nécessaires à la fabrication de sa coquille. Or, les ions carbonate contenant les isotopes lourds de l'oxygène $\left({ }^{18} \mathrm{O}\right)$ ne précipitent pas à la même vitesse que ceux comportant les isotopes légers $\left({ }^{16} \mathrm{O}\right)$, et la différence de vitesse de précipitation entre les isotopes lourds et légers est d'autant plus grande que la température de l'eau est basse. La composition isotopique d'un carbonate de calcium d'origine biologique

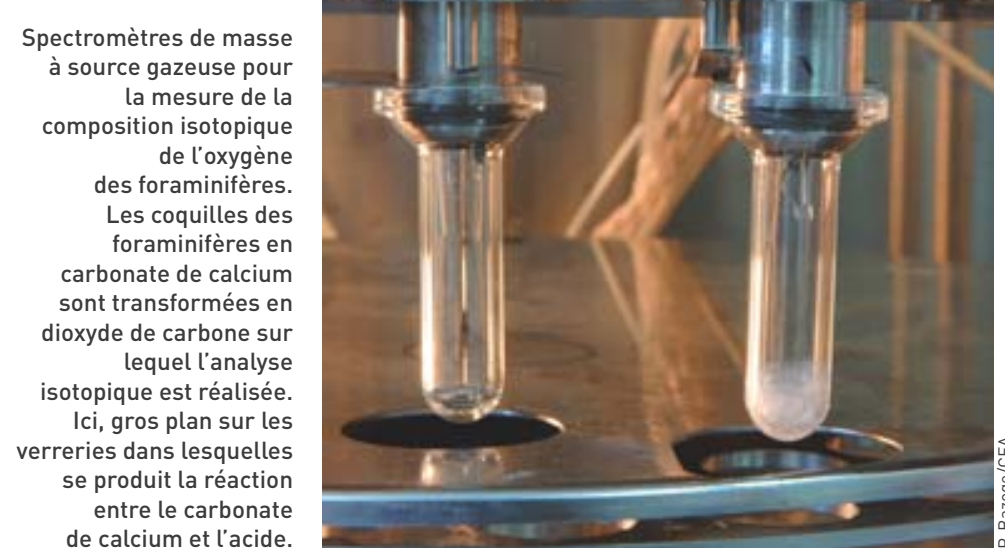

constitue donc un thermomètre pour l'eau dans laquelle il s'est développé. Il faut toutefois souligner que cette composition isotopique dépend également de celle de l'eau dans laquelle le carbonate a précipité. La mesure physique consiste donc à transformer le carbonate de calcium en dioxyde de carbone $\left(\mathrm{CO}_{2}\right)$ sur lequel la composition isotopique de l'oxygène sera mesurée.

C'est grâce aux travaux pionniers du chimiste américain Harold Clayton Urey en 1947 que la paléoclimatologie isotopique a pu se développer. Cela a sans conteste joué un rôle majeur dans la compréhension des mécanismes climatiques naturels qui impliquent l'océan, l'atmosphère, la cryosphère et la biosphère. Deux exemples de résultats acquis au Laboratoire des sciences du climat et de l'environnement (LSCE) sont détaillés ci-dessous.

\section{Comprendre l'impact de débâcles d'icebergs sur la circulation océanique}

Les études effectuées à partir de sédiments marins et de glaces polaires ont montré que le climat de la dernière période glaciaire, il y a entre 60000 ans et 10000 ans, était ponctué d'épisodes très froids, extrêmement rapides à l'échelle des temps géologiques, et d'impact au moins hémisphérique. De tels événements, appelés événements de Heinrich, sont dus à des débâcles massives d'icebergs dans l'océan Atlantique Nord, entre 40 et $60^{\circ} \mathrm{N}$ environ (figure 1). Leur enregistrement dans les sédiments marins se traduit par la présence de niveaux riches en cailloux de toutes tailles et de toutes natures pétrographiques, au milieu d'un sédiment généralement riche en micro-organismes carbonatés. Ces débâcles d'icebergs ont provoqué en fondant un énorme apport d'eau douce, un ralentissement du transport des eaux chaudes et salées des basses latitudes vers les hautes latitudes par la dérive Nord Atlantique et un refroidissement des eaux de surface de l'Atlantique Nord. Les carottes de sédiments marins ont permis de dresser une cartographie de l'impact isotopique d'un événement de Heinrich sur les eaux de surface et d'en quantifier l'influence en termes de température et de salinité. Les températures des eaux de surface ont diminué en moyenne de 2 à $4{ }^{\circ} \mathrm{C}$ pendant ces débâcles et leur salinité d'environ 0,5\%o. Cette diminution conjuguée de la température et de la salinité a modifié la densité des eaux de surface, affectant ainsi la circulation océanique à une échelle bien plus large que le seul océan Atlantique Nord.

Si la géochimie isotopique a renseigné sur l'amplitude de la chute de salinité, la durée de ces événements et leur intensité en termes de volume de glace impliqué étaient toujours très mal estimées (de 0,1 à 10 millions de $\mathrm{km}^{3}$, en 10 ans ou en plus de 1000 ans). La simulation numérique du transport des isotopes de l'eau dans des expériences de débâcles d'icebergs a permis d'apporter des éléments de réponse aux questions relatives à la durée et à l'intensité de la débâcle. Or, ces deux paramètres sont fondamentaux pour quantifier les seuils de réponse de la circulation thermohaline dans une décharge d'eau douce. Pour cela, les chercheurs du LSCE ont utilisé un modèle de complexité intermédiaire 


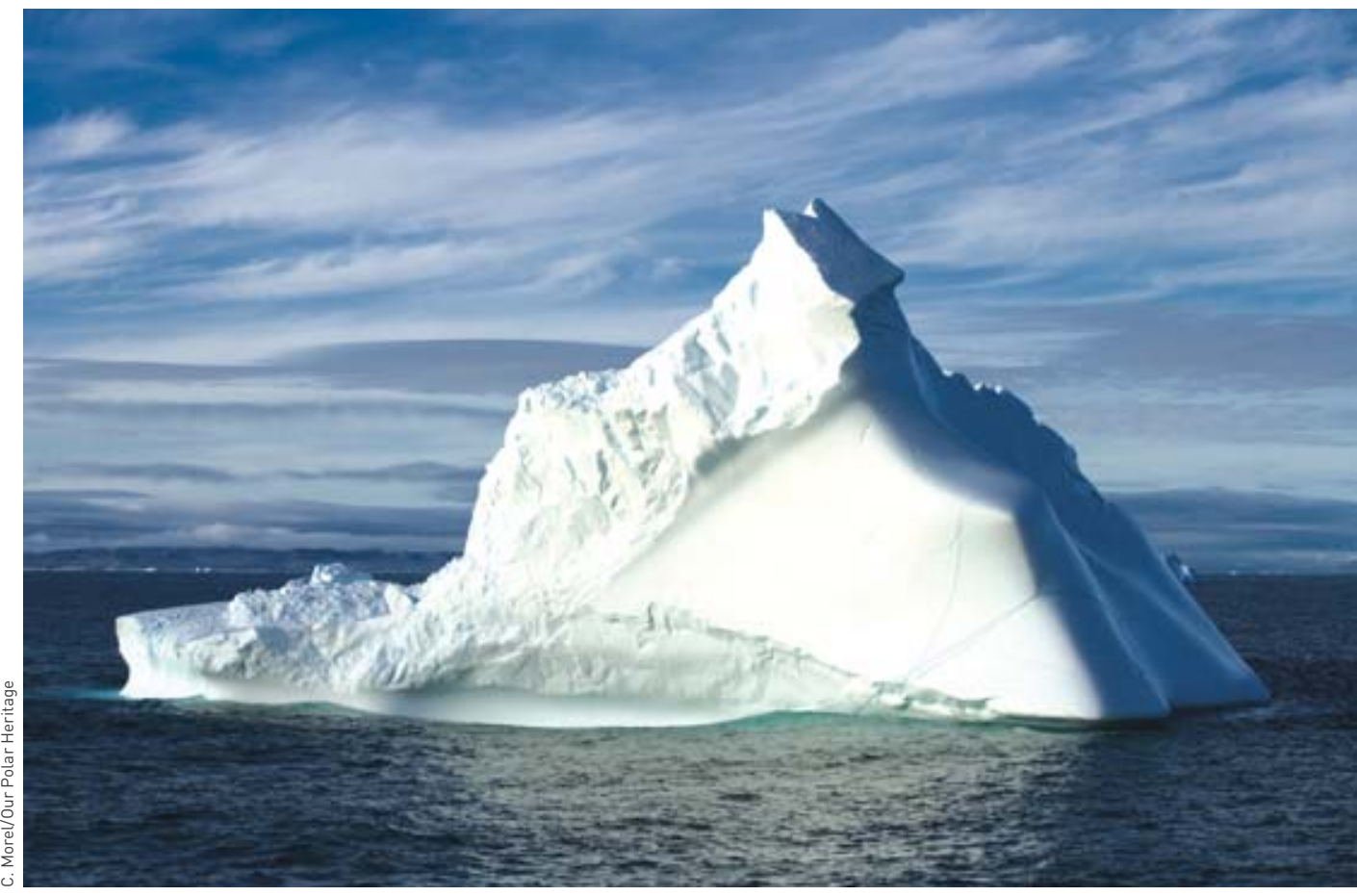

L'analyse de carottes de glaces polaires et de sédiments marins a mis en évidence des événements climatiques froids et extrêmement rapides survenus au cours de la dernière période glaciaire. Ils sont dus à des débâcles massives d'icebergs qui ont amené d'énormes quantités d'eau douce dans l'Atlantique Nord, perturbant fortement la circulation océanique.

(EMIC) pour reproduire une débâcle d'icebergs survenue il y a 40000 ans, en modifiant sa durée et son intensité, et ont déterminé les simulations ressemblant le plus aux données mesurées dans les sédiments marins ${ }^{(1)}$. La meilleure analogie entre expériences numériques et données isotopiques est obtenue pour des durées de débâcle d'icebergs de $200 \pm 100$ ans et un flux d'eau douce de l'ordre de $0,25 \cdot 10^{6} \mathrm{~m}^{3} / \mathrm{s}$, soit un total de 1,5 million de $\mathrm{km}^{3}$ de glace dont la fonte aura fait monter le niveau de la mer d'environ $3 \mathrm{~m}$. L'extrême brièveté de cet événement était inattendue en raison de son énorme impact sur le climat global.

\section{Appréhender les mécanismes possibles de déstabilisation des calottes glaciaires}

Plus proche du climat que nous connaissons actuellement, la dernière période interglaciaire précédant la nôtre, survenue il y a environ entre 129000 ans et 118000 ans, a été riche d'enseignement. Les archives géologiques, qu'elles soient marines, glaciaires ou continentales, ont permis de montrer que les températures de l'air et de l'océan de surface durant cette période étaient en moyenne de 2 à $5{ }^{\circ} \mathrm{C}$ plus chaudes que celles de notre interglaciaire, appelé Holocène. Le niveau marin était de l'ordre de 4 à $6 \mathrm{~m}$ plus haut que l'actuel, à cause en particulier de la fonte partielle des calottes de glace continentales présentes au Groenland et en Antarctique de l'Ouest. Les calottes de glace n'ont pas toutes la même sensibilité aux changements de température: en Arctique, la dernière période interglaciaire était assez chaude pour réduire d'environ $50 \%$ la taille de la calotte groenlandaise. En revanche, en Antarctique, la température de l'air est très basse et la calotte Antarctique de l'Ouest, dont la

(1) D. Roche, D. Paillard and E. Cortijo, "Constraints on the duration and freshwater release of Heinrich event 4 through isotope modelling", Nature, 432, p. 379-382, 2004.

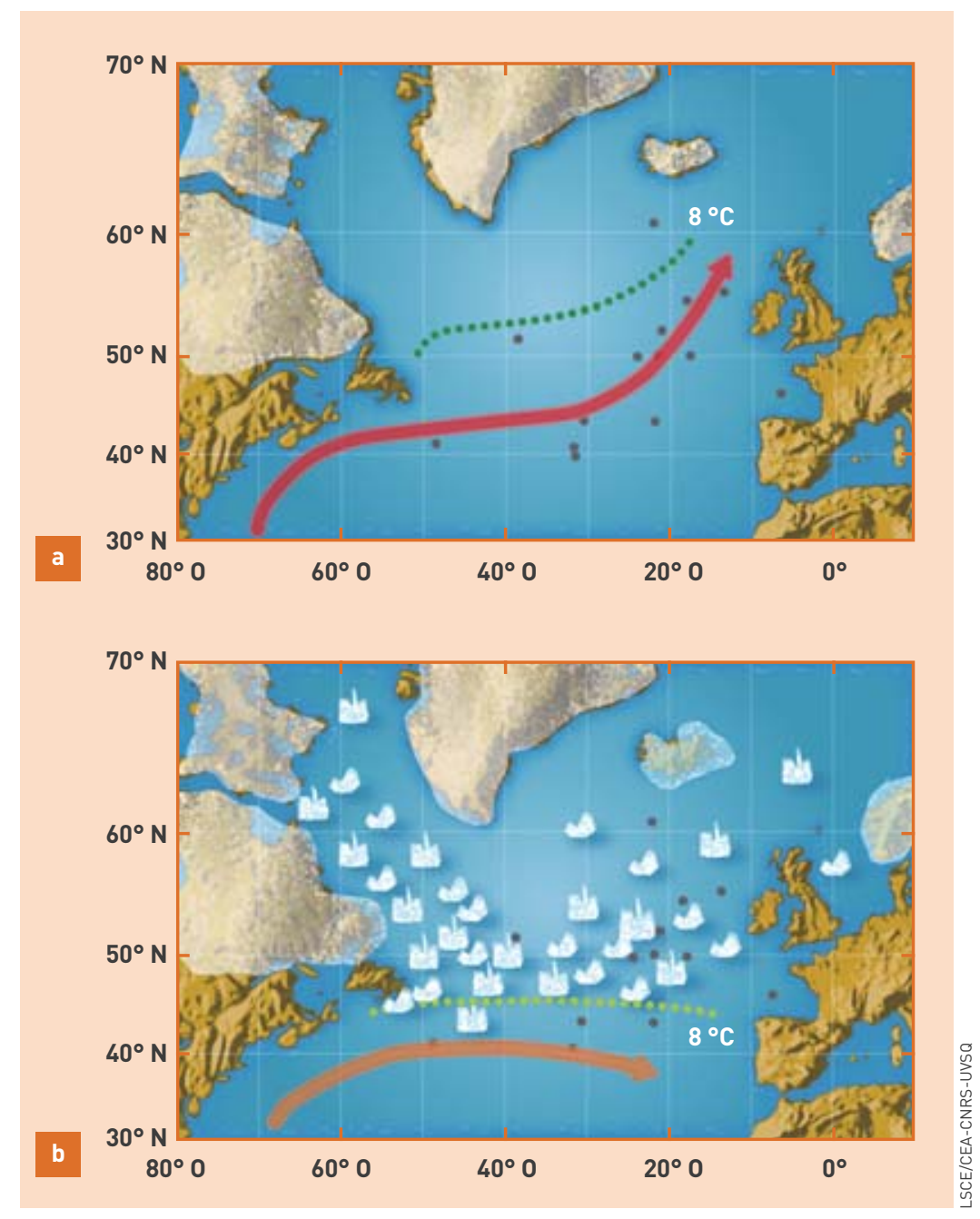

Figure 1.

Les mécanismes des événements de Heinrich. En a, température en période glaciaire, avant l'événement de débâcle. En b, impact d'une débâcle d'icebergs sur les températures des eaux de surface. Le retour à la normale est observé après la disparition des eaux de fonte d'icebergs. Les flèches correspondent à la dérive Nord Atlantique en mode non perturbé (en rouge) et en mode perturbé (en orange) et les lignes en pointillés à l'isotherme $8^{\circ} \mathrm{C}$. 
